guidance for final year registrars, and opening the scheme to them prior to commencement of their substantive posts, we anticipate that these changes will bring further benefits to those in the mentoring programme.

\section{P190 BAREBACKING: OPINIONS OF HIV NEGATIVE MEN WHO HAVE SEX WITH MEN}

doi:10.1136/sextrans-2012-050601c.190

${ }^{1} \mathrm{M}$ Grundy-Bowers, ${ }^{*}{ }^{2} \mathrm{~A}$ Black. ${ }^{1}$ City University London, London, UK; ${ }^{2}$ Imperial College Healthcare NHS Trust, London, UK

Background Men who have sex with men (MSM) remain disproportionately affected by HIV and sexual infections. 2010 saw the highest number of new UK HIV diagnoses, acquired predominately through condomless anal sex (CAS) with an estimated $67 \%$ acquired in relationships. To reduce risk taking behaviour, a deeper understanding of what influences MSM not to use condoms is required. Aim To explore the opinions and rationales for CAS in HIV negative MSM.

Methods MSM were targeted, via gay press and leafleting, to complete an online questionnaire exploring issues around CAS. Data were collected from November 2010 to October 2011 following ethical approval. Responses from HIV negative MSM were reviewed and themed to quantify opinions and motivations for CAS in this cohort.

Results Data are drawn from a larger study. A total of 158 males met the criteria; $73.4 \%(n=116)$ did not identify themselves as a barebacker, the remainder did. There was a mean age of 35.4 (range 18-72), with the majority being White British (48.7\%, n=77). All participants had engaged in unprotected anal sex. Barebacking was identified as contextual (eg, only in relationship) by 36 or behavioural (eg, I bareback) by 28 respondents. Relationships (79, 50\%), trust $(44,27.8 \%)$ and infection screening $(42,26.6 \%)$ featured most frequently as personal reasons for engaging in CAS. Alcohol (63, $39.9 \%)$, physical sensation $(58,36.7 \%$ ) and the thrill of the risk (53, $33.5 \%$ ) were the most common opinions on why others had unprotected sex.

Conclusions CAS remains a complex issue and the definition of "barebacker" varies. Perceptions why others engaged in CAS have more negative connotations, however individual rationales for engaging in CAS predominantly focussed around love and relationships. Given the significant number of MSM that acquire HIV in relationships there is a clear need to maintain safer sex messages that are contextualised for those in relationships to observe the principles of negotiated safety.

\section{P191 DEMONSTRATING PERFORMANCE OF A LOW-COST ULTRA-RAPID PCR ASSAY FOR TRICHOMONAS VAGINALIS WITH POINT-OF-CARE APPLICATIONS}

doi:10.1136/sextrans-2012-050601c.191

D M Pearce, ${ }^{*}$ D N Styles. Atlas Genetics Ltd.

Objectives We have developed a novel Point-of-Care (PoC) system, Velox, comprising an assay-specific cartridge and instrument with a turnaround time of $25 \mathrm{~min}$. The system has been developed for Chlamydia trachomatis and Chlamydia/Gonorrhoea. We have now developed a Trichomonas vaginalis (TV) test suitable for integration onto the cartridge. The assay utilises a novel electrochemical detection method to demonstrate low copy number amplification and detection of TV in $<25 \mathrm{~min}$.

Methods The method employs prototype PCR cartridges in conjunction with an ultra-rapid thermocycler. All reagents necessary to perform the extraction, amplification and detection are deposited into the cards and air dried at the point of manufacture. A sample is added to the card and DNA extracted from the sample. The resulting eluate reconstitutes dried PCR reagents and PCR is performed using rapid thermocycling. Amplified target is detected using electrochemically-labelled TV target-specific probes and a double-stranded DNA-specific exonuclease to release the electrochemical label. Released label is read by applying a voltage to a screen printed carbon electrode and at a known oxidation potential the label is oxidised producing a measurable current.

Results Analytical sensitivity of the TV assay was evaluated by testing dilutions of the organism in the presence of Internal Control (IC) DNA. The results show a TV limit of detection of 50 copies when co-extracted, amplified in duplex and detected electrochemically with the IC DNA. Tests on the reagents dried into the device showed stability for 18 months when stored at ambient temperature $\left(20-25^{\circ} \mathrm{C}\right)$

Conclusions The results show that in conjunction with the instrument, the TV assay could be used to perform ultra-rapid PCR with no user intervention after sample addition. This allows minimallytrained staff to carry out the assay in $<25 \mathrm{~min}$, meeting the needs of a PoC device.

\section{P192 WHAT CAREER CHOICES DO TRAINEES MAKE AFTER ATTENDING THE GU MEDICINE TASTER?}

doi:10.1136/sextrans-2012-050601c.192

S Patel, ${ }^{*}$ R Lau. St George's Hospital, London, UK

Background The GUM taster (running annually in London since 2009) is a 2-day educational programme for junior doctors before they apply for specialist training. It is popular and oversubscribed, receiving excellent feedback. We sought to investigate the career choices made by trainees who had attended previous GUM tasters.

Methods All trainees who had attended one of three previous GUM tasters were contacted about their career progression. Four questions were asked, covering: (1) current specialty, (2) current year of training, (3) career choices/plan, (4) reasons for choice of specialty.

Results Trainees were contacted by email and telephone, response rate $86 \%(90 / 105)$. Trainees currently in specialty or GP training $(52 \%, 47 / 90)$ are listed below: The remaining trainees (43/ 90) were in: non-training clinical posts/working abroad (21), still in F2/CT1/CT2 (19), clinical fellowships (2) or had left medicine (1). Of these, $37 \%(16 / 43)$ planned to apply to GUM as their first choice specialty; with $26 \%$ (11/43) planning to apply for GP training. $71 \%$ $(15 / 21)$ of trainees planning a career as a GP wanted to continue with GU as a specialist interest. Reasons given for choosing GU medicine included interest, work-life balance, opportunities for research/work abroad, lively colleagues, diverse patients and avoiding general medicine (see abstract P192 table 1).

Discussion $37 \%$ (33/90) of those attending our GU Tasters were either already in GU training or considering applying for GU as their first choice specialty. In 2011, the Taster was successfully organised in London and in the Midlands (for the first time). The course has allowed trainees to be better informed about the specialty and has highlighted GU medicine as a popular alternative or additional career option for many. 